NASA Contractor Report 198354

ICOMP-95-11; AIAA-95-2167

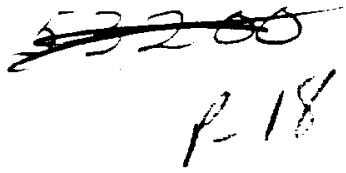

\title{
An Assessment of Artificial Damping Models for Aeroacoustic Calculations
}

M. Ehtesham Hayder

Institute for Computational Mechanics in Propulsion

Lewis Research Center

Cleveland, Ohio

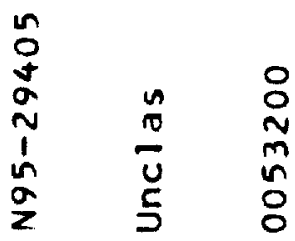

June 1995

$\sum_{0}^{m}$

Prepared for

Lewis Research Center

Under Contract NCC3-370

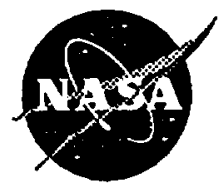

National Aeronautics and

Space Administration
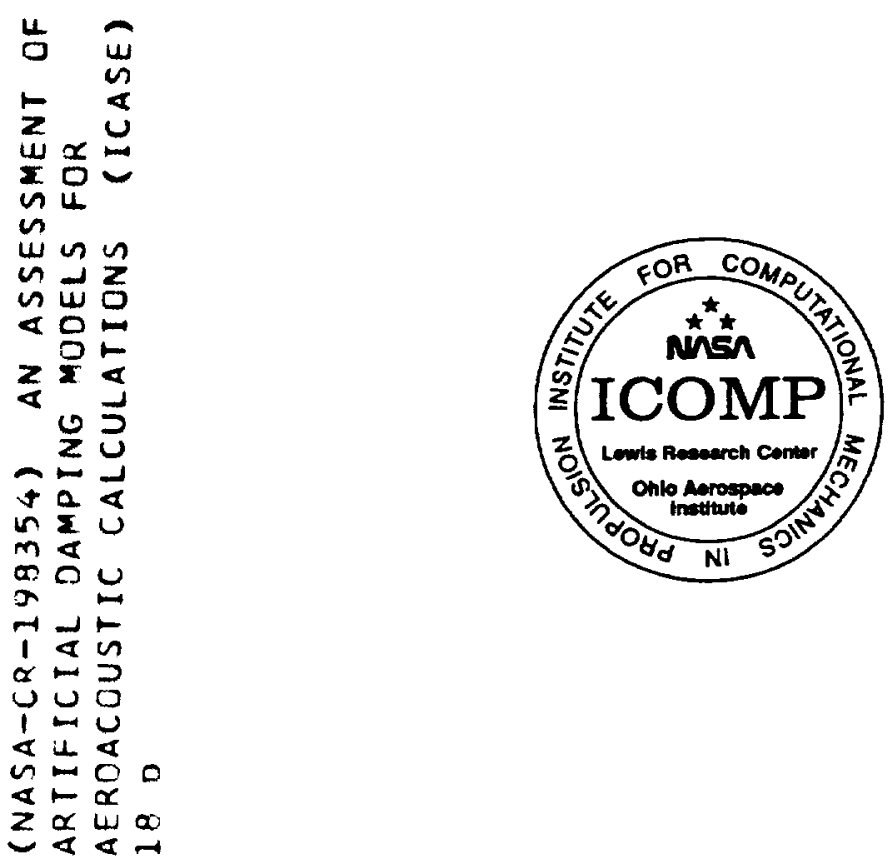


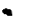




\title{
An Assessment of Artificial Damping Models for Aeroacoustic Calculations
}

\author{
M. Ehtesham Hayder \\ Institute for Computational Mechanies in Propulsion \\ NASA Lewis Research Center \\ Cleveland, OH 44135
}

\begin{abstract}
We present a study of the effect of artificial dissipation models on nonlinear wave computations using a few high order schemes. Our motivation is to assess the effectiveness of artificial dissipation models for their suitability for aeroacoustic computations. We solve three model problems in one dimension using the Euler equations. Initial conditions are chosen to generate nonlinear waves in the computational domain. We examine various dissipation models in central difference schemes such as the Dispersion Relation Preserving (DRP) scheme and the standard fourth and sixth order schemes. We also make a similar study with the fourth order MacCormack scheme due to Gottieb and Turkel.
\end{abstract}

KEY WORDS: Artificial dissipation, High order schemes, Computational aeroacoustics

\section{Introduction}

Very high numerical accuracy is essential in aeroacoustics computations. To get such accuracy, one should use high order schemes with very little numerical dispersion and dissipation. Numerical solutions with high order schemes are usually very good in flows without shocks. However, because of inadequate numerical dissipation a high order scheme may generate spurious oscillations in presence of shocks or steep gradients. In this study we consider computations of flows with very sharp

Copyright (C)1995 by the American Institute of Aeronautics and Astronautics, Inc. No copyright is asserted in the United States under Title 17, U.S. Code. The U.S. Government has a royaltyfree license to exercise all rights under the copyright claimed herein or Governmental purposes. All other rights are reserved by the copyright owner. gradients. Such flow conditions may appear in supersonic jet noise computations. All basic schemes used in this study are formally at least fourth order accurate in space. We augment these basic schemes with various artificial dissipation models and evaluate these artificial dissipation models. Tam and his colleagues specifically designed basic scheme for aeroacoustic calculations and it is known as the Dispersion Relation Preserving (DRP) scheme $1,2,3$. The DRP is a central finite difference scheme. We also consider the standard fourth and the sixth order central difference schemes and the fourth order MacCormack scheme due to Gottlieb and Turkel ${ }^{4}$. The fourth order MacCormack scheme has been tested for accuracy in aeroacoustic applications 5,6 and is extensively used, e.g. $5,7,8,9,10$.

In their study of the DRP schemes for nonlinear wave computations, Tam and his colleagues discovered that the wavelengths of spurious oscillations are concentrated in a narrow band in short wave range. They formulated a selective artificial damping model to suppress such oscillations and used it for computation of nonlinear pulses ${ }^{3}$. There are many other dissipation models which are also shown to be effective in flow computations. One such dissipation model was proposed by Jameson et al. 11. This model is widely used in aerodynamic calculations. Turkel ${ }^{12}$ extended this model to a set of matrix-valued coefficients to give appropriate viscosity for each wave component. In this study, we will consider these dissipation models and also the model proposed by MacCormack and Baldwin ${ }^{13}$.

In the next section we give the governing equations. Descriptions of the basic schemes and artificial dissipation models are presented in sections 3 and 4 respectively. We give our test problems in section 5 and discuss results in section 6 . 


\section{Governing Equations}

We solve the one dimensional Euler equations written in the following form

$$
Q_{t}+f_{x}=0
$$

where

$$
\begin{gathered}
Q=\left(\begin{array}{c}
\rho \\
\rho u \\
E
\end{array}\right) \\
f=\left(\begin{array}{c}
\rho u \\
\rho u^{2}+p \\
\rho u H
\end{array}\right) \\
p=(\gamma-1)\left(E-\frac{1}{2} \rho u^{2}\right) \\
H=\frac{E+p}{\rho}
\end{gathered}
$$

where $\rho, \mathrm{u}, \mathrm{P}, \gamma, \mathrm{E}$ and $\mathrm{H}$ are the density, velocity, pressure, ratio of specific heats, total energy and enthalpy respectively.

\section{Basic Schemes}

We consider a high order MacCormack scheme along with a few central difference schemes. The MacCormack scheme nses forward and backward differences in predictor and corrector sweeps. All basic schemes presented in this study can be written in conservative discrete form. Such conservation is very important for flows with shocks and is illustrated by the fundamental theorem of Lax and Wendroff ${ }^{14}, 15$.

\subsection{The DRP and Central Schemes}

Tam and Webb ${ }^{1}$ developed a class of high order scheme known as the Dispersion Relation Preserving (DRP) scheme for computational acoustics. They used central differencing for spatial discretiration. The coefficients of their spatial discretization scheme were chosen by requiring the Fourier transform of the finite difference scheme be a close approximation to that of the partial derivatives. Tam and Webb used a seven point stencil for fourth order spatial accuracy. They proposed a multilevel finite difference time integration scheme. Coefficients of this time integration algorithm were chosen so that the Laplace transform of the finite difference scheme is a good approximation of the partial derivative. Their scheme can be written as

$$
K_{i}^{n}=-\frac{1}{\Delta x} \sum_{j=-3}^{3} a_{j} f_{i+j}^{n}
$$

$$
\begin{gathered}
Q_{i}^{n+1}=Q_{i}^{n}+\Delta t \sum_{j=0}^{3} b_{j} K_{i}^{n-j} \\
a_{0}=0 ; \quad a_{1}=-a_{-1}=0.79926643 \\
a_{2}=-a_{-2}=-0.18941314 \\
a_{3}=-a_{-3}=0.02651995
\end{gathered}
$$

$$
\begin{array}{ll}
b_{0}=2.30255809 ; & b_{1}=-2.49100760 \\
b_{2}=1.57434093 ; & b_{3}=-0.38589142
\end{array}
$$

Above DRP scheme is formally fourth order accurate in space and third order accurate in time. In a later study, Tam and Shen ${ }^{2}$ revised the values of the coefficients $a_{j}$ to obtain a better overall numerical wave characteristics for a seven point stencil. Revised $a_{j}$ coefficients are

$$
\begin{gathered}
a_{1}=-a_{-1}=0.770882380518 \\
a_{2}=-a_{-2}=-0.166705904415 \\
a_{3}=-a_{-3}=0.020843142770
\end{gathered}
$$

For all computations with the DRP scheme in this paper, we used the above set of values for $a_{j}$. This DRP scheme has a seven point stencil and formal fourth order spatial accuracy. The coefficients $a_{j}$ in the standard fourth order central difference scheme are

$$
a_{0}=0 ; \quad a_{1}=-a_{-1}=\frac{2}{3} ; \quad a_{2}=-a_{-2}=-\frac{1}{12}
$$

The stencil is five points wide, i.e., $a_{3}=a_{-3}=0$. Similarly the standard sixth order central difference scheme on a seven point stencil is

$$
\begin{gathered}
a_{0}=0 ; \quad a_{1}=-a_{-1}=\frac{3}{4} ; \quad a_{2}=-a_{-2}=-\frac{3}{20} \\
a_{3}=-a_{-3}=\frac{1}{60}
\end{gathered}
$$


For time integration of the DRP, the standard fourth and the sixth order central difference schemes we use the algorithm given in eq. 3.1b. Thus the formal temporal accuracy for these calculations is third order.

\subsection{High order MacCormack Scheme}

Gottlieb and Turkel ${ }^{4}$ extended the standard MacCormack scheme (2nd order accurate in both space and time) to a spatially fourth order accurate scheme. In this study we will refer this scheme as the fourth order MacCormack scheme. This scheme has a predictor and a corrector stage and for one dimensional computations may be written as:

the predictor step with forward differences is

$$
\bar{Q}_{i}=Q_{i}^{n}+\frac{\Delta t}{6 \Delta x}\left\{7\left(f_{i+1}^{n}-f_{i}^{n}\right)-\left(f_{i+2}^{n}-f_{i+1}^{n}\right)\right\}
$$

and the corrector step with backward differences is

$$
\begin{gathered}
Q_{i}{ }^{n+1}=\frac{1}{2}\left[\bar{Q}_{i}+Q_{i}^{n}\right. \\
\left.+\frac{\Delta t}{6 \Delta x}\left\{7\left(\vec{f}_{i}-\bar{f}_{i-1}\right)-\left(\bar{f}_{i-1}-\bar{f}_{i-2}\right)\right\}\right]
\end{gathered}
$$

This scheme is second order accurate in time and becomes fourth order accurate in the spatial derivatives when alternated with symmetric variants 4 , 16. We define $L_{1}$ as a one dimensional operator with a forward difference in the predictor and a backward difference in corrector. Its symmetric variant $L_{2}$ uses a backward difference in the predictor and a forward difference in the corrector. Therefore to ensure the fourth order spatial accuracy, the sweeps are arranged in our computations as

$$
\begin{gathered}
Q^{n+1}=L_{1} Q^{n} \\
Q^{n+2}=L_{2} Q^{n+1}
\end{gathered}
$$

In this study when we add any disspation model in this scheme (except the MacCormack-Baldwin model in \$4.2) we modify only the corrector step as

$$
\begin{gathered}
Q_{i}^{n+1}=\frac{1}{2}\left[\bar{Q}_{i}+Q_{i}^{n}\right. \\
\left.+\frac{\Delta t}{6 \Delta x}\left\{7\left(\bar{f}_{i}-\bar{f}_{i-1}\right)-\left(\bar{f}_{i-1}-\bar{f}_{i-2}\right)\right\}\right]+\Delta t D_{i}
\end{gathered}
$$

where $D_{i}$ is the artificial dissipation at location $i$. Alternatively, one can also add dissipation in both predictor and corrector steps as in the case of MacCormack Baldwin model ( $(4.2)$.

\section{Artificial Dissipation Models}

\subsection{Tam's Model}

The basic DRP scheme (\$3.1) has very little numerical disspation. In order to compute nonlinear waves with this scheme, Tam and his colleagues 2,3 formulated an artificial selective damping term to be added to the basic algorithm. Thus, the eq. 3.1a becomes

$$
\begin{gathered}
K_{i}^{n}=-\frac{1}{\Delta x} \sum_{j=-3}^{3} a_{j} f_{i+j}^{n}+D_{i}^{n} \\
D_{i}^{n}=-\frac{u_{i}^{\prime}}{\Delta x R_{,}} \sum_{j=-3}^{3} c_{j} Q_{i+j}^{n}
\end{gathered}
$$

where $u_{i}^{s}=\left|u_{i}^{\max }-u_{i}^{\min }\right|$ is the difference between the maximum and the minimum velocity in the computational stencil centered at location $i$. The coefficients $c_{j}$ are determined by requiring the damping function be a close approximation to a Gaussian function. Tam and Shen ${ }^{2}$ recommended following numerical parameters after extensive numerical experiments:

$$
\begin{array}{ll}
c_{0}=0.327698660846 ; & c_{1}=-0.235718815308 \\
c_{2}=0.086150669577 ; & c_{3}=-0.014281184692
\end{array}
$$

The damping function $(D)$ is an even function, i.e., $c_{-j}=c_{j}$. Their recommended value for the stencil Reynolds number $\left(R_{s}\right)$ is 0.1 . Recently Tam ${ }^{17}$ used the stencil Reynolds number equal to 0.05 in his computation of the wave equation. We would like to point out that Tam and his colleagues formulated this dissipation model to specifically maintain the correct propagation characteristics of waves and damp short waves generated by numerical solution, rather than to maintain high order of accuracy in the sense of expanded Taylor series. Thus this model should yield good results related to wave propagation characteristics.

\subsection{MacCormack-Baldwin Model}

MacCormack and Baldwin ${ }^{13}, 18$ devised a dissipation model for the MacCormack scheme. Thus, the modified flux becomes 


$$
\hat{f}_{i}=f_{i}+\epsilon\left(\left|u_{i}\right|+c\right) \frac{\left|p_{i+1}-2 p_{i}+p_{i-1}\right|}{p_{i+1}+2 p_{i}+p_{i-1}} d Q_{i}
$$

where $d Q_{i}$ is a forward difference (i.e., $Q_{i+1}-Q_{i}$ ) in the backward sweep and vice versa. The physical flux and the speed of sound are $f$ and $c$ respectively. The modified scheme with artificial viscosity is obtained by replacing $f$ by $\hat{f}$ in the basic scheme (i.e. in eq. 3.2a and 3.2b for the fourth order MacCormack scheme). The parameter $\epsilon$ should be between 0 and $\frac{1}{2}$ for stability. In our tests we use $\epsilon=\frac{1}{4}$.

\subsection{JST Model}

Jameson, Schmidt and Turkel ${ }^{11}$ proposed an artificial dissipation model where the convective flux is modified as

$$
\hat{f}_{i+\frac{1}{2}}=f_{i+\frac{1}{2}}-d_{i+\frac{3}{2}}
$$

where

$$
d_{i+\frac{1}{2}}=\lambda_{i+\frac{1}{3}}\left[\epsilon_{i+\frac{1}{2}}^{(2)} d W_{i}-\epsilon^{(4)}\left(d W_{i+1}-2 d W_{i}+d W_{i-1}\right)\right]
$$

$$
\begin{gathered}
d W_{i}=W_{i+1}-W_{i} \\
\epsilon_{i+\frac{2}{2}}^{(2)}=\kappa^{(2)} \max \left(\nu_{i+1}, \nu_{i}\right) \\
\epsilon_{i+\frac{1}{2}}^{(4)}=\max \left(0,\left[\kappa^{(4)}-\epsilon_{i+\frac{1}{2}}^{(2)}\right]\right) \\
\nu_{i}=\frac{\left|p_{i+1}-2 p_{i}+p_{i-1}\right|}{p_{i+1}+2 p_{i}+p_{i-1}} \\
W=Q+[0,0, p]^{T}
\end{gathered}
$$

$\kappa^{(2)}=\frac{1}{4}, \kappa^{(4)}=\frac{1}{258}$ and $\lambda$ is a scaling factor. In this study we use $\lambda=|u|+c$. The second difference term adds dissipation near the shock while the fourth difference term damps high-frequency modes and reduces to zero near the shock. We will refer this model as the JST dissipation model in this paper. The damping function $\left(D_{i}\right)$ for this model is as follows:

$$
D_{i}=\frac{\left[d_{i+\frac{2}{2}}-d_{i-\frac{2}{2}}\right]}{\Delta x}
$$

\subsection{Turkel's Matrix-Valued Model}

Turkel ${ }^{12}$ proposed a matrix scaling instead of scalar scaling in the JST dissipation model. This matrix dissipation model was tested by Jorgenson and Turkel ${ }^{19}$. One major objective of this model is to reduce the amount of artificial dissipation for slower waves. Thus the scaling parameter $\lambda$ in eq. 4.3 becomes a matrix. Details of this matrix are given in the above cited references. We implemented this model as follows:

$$
D_{i}=\Lambda \frac{\left(\Delta Q_{i}-\Delta Q_{i-1}\right)}{\Delta x}
$$

$$
\begin{aligned}
& \Lambda=\lambda_{3} I+\left(\frac{\lambda_{1}+\lambda_{2}}{2}-\lambda_{3}\right)\left[\frac{\gamma-1}{c^{2}} G_{1}+G_{2}\right] \\
& +\frac{\lambda_{1}-\lambda_{2}}{2 c}\left[G_{3}+(\gamma-1) G_{4}\right] \\
& G_{1}=\left(\begin{array}{c}
R_{1} \\
u R_{1} \\
H R_{1}
\end{array}\right) \\
& G_{2}=\left(\begin{array}{c}
0 \\
R_{2} \\
u R_{2}
\end{array}\right) \\
& G_{3}=\left(\begin{array}{c}
R_{2} \\
u R_{2} \\
H R_{2}
\end{array}\right) \\
& G_{4}=\left(\begin{array}{c}
0 \\
R_{3} \\
u R_{1}
\end{array}\right) \\
& R_{1}=\left(\frac{u^{2}}{2},-u, 1\right) \\
& R_{2}=(-u, 1,0) \\
& R_{\mathbf{s}}=\left(\boldsymbol{u}^{2},-u, 1\right)
\end{aligned}
$$

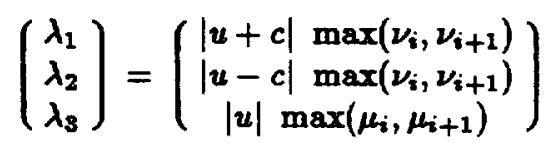

$$
\begin{aligned}
& \nu_{i}=\frac{\left|p_{i+1}-2 p_{i}+p_{i-1}\right|}{p_{i+1}+2 p_{i}+p_{i-1}} \\
& \mu_{i}=\frac{\left|T_{i+1}-2 T_{i}+T_{i-1}\right|}{T_{i+1}+2 T_{i}+T_{i-1}}
\end{aligned}
$$




$$
\begin{gathered}
d Q_{i}=Q_{i+1}-Q_{i} \\
\epsilon^{(2)}(j)=\kappa^{(2)} \lambda_{j} \\
\epsilon^{(4)}(j)=\max \left[0, \kappa^{(4)}-\epsilon^{(2)}(j)\right]
\end{gathered}
$$

$\Delta Q_{i}(j)=\epsilon_{i}^{(2)}(j) d Q_{i}+\epsilon_{i}^{(4)}(j)\left(d Q_{i+1}-2 d Q_{i}+d Q_{i-1}\right)$

where $\mathrm{T}$ is temperature, parameters $\kappa^{(2)}$ and $\kappa^{(4)}$ are same as in the JST model.

\section{Test Problems}

We consider three test problems to evaluate various dissipation models in the basic schemes. The first two problems were previously used to evaluate various algorithms in the ICASE/LaRC Workshop on Benchmark Problems in Computational Aeroacouties $^{20}$. In addition we consider Lax's shock tube problem.

\subsection{Test Problem 1}

In this problem a initial pulse steepens with time. Initial conditions are given as:

$$
\begin{gathered}
u=\frac{1}{2} e^{-\left(\frac{g}{b}\right)^{2}(\ln 2)} \\
p=\frac{1}{\gamma}\left(1+\frac{\gamma-1}{2} u\right)^{\frac{2 \gamma}{\gamma-1}} \\
\rho=\left(1+\frac{\gamma-1}{2} u\right)^{\frac{3}{\gamma-1}}
\end{gathered}
$$

The computational domain is $-50<x<350$.

\subsection{Test Problem 2}

This is similar to a standard shock tube problem. There is a very sharp gradient instead of a discontinuity in the initial condition. Initial conditions are

$$
\begin{array}{lll}
u=0 & \\
p=4.4 & x<-2 \\
p=2.7+1.7 \cos \left[\frac{(x+2) \pi}{4}\right], & -2 \leq x \leq 2 \\
p=1 . & x>2 \\
\rho= & (\gamma p)^{\frac{1}{\gamma}} &
\end{array}
$$

The computational domain is $-100<x<100$.

\subsection{Test Problem 3 (Lax's problem)}

This is a Riemann (shock tube) problem used by $\operatorname{Lax}^{21}$. Initial conditions are

$$
\begin{aligned}
& \mathrm{u}=.698, \mathrm{p}=3.528, \rho=.445 \text { for } 0<x<.5 \\
& \mathrm{u}=0, \mathrm{p}=.571, \rho=.5 \text { for } .5<x<1
\end{aligned}
$$

The computational domain is $0<x<1$.

\section{Results}

One uses artificial dissipation to eliminate spurious numerical oscillations in computed flows with shocks or steep gradients. Too little dissipation may not be enough to remove spurious oscillations, while too much dissipation will smear of the solution profile and destroy the accuracy of the computed solution. Therefore one should try to add appropriate amount of dissipation to a basic scheme. Our primary goal in this study is to examine the effectiveness of a few artificial dissipation models. We tried to keep the model constants same as in cited references and did not attempt to optimize any constant. We tested these dissipation models in a few basic schemes. A comparison of two such schemes, namely the DRP and the fourth order MacCormack scheme (withont dissipation) for the first two test problems was presented in an earlier study ${ }^{22}$. Here we also consider the standard fourth and sixth order central difference schemes. For all our computations reported in this paper we used $\mathbf{4 0 1}$ grid points and the characteristic boundary condition at the boundaries. In the first test problem, an initial pulse steepens with time and nonlinear effects become very important. In Figure 1, we show the evolution of the density profile for this test problem. These computations were made using the DRP scheme with the Tam dissipation model with the stencil Reynolds number $\left(R_{a}\right)$ equal to 0.05 . We observe that the initial profile steepens quickly and at $t=100$ the leading edge becomes very sharp. We chose this time level to evaluate the damping models and the basic schemes for computations of the test problem 1. All computations with the central difference schemes (the DRP, standard 4th and 6th order central difference scheme) presented in this paper were done with CFL number equal to $\frac{1}{20}$. In Figure 2, we show density profiles computed using the DRP scheme at $t=100$. There is excessive amount of oscillations in the computed solution when no artificial dissipation is used. Both the Tam dissipa- 
tion model $\left(R_{t}=0.1\right)$ and Turkel's matrix valued disspation model eliminated most of the oscillations. We used $\kappa^{(2)}=4$ and $\kappa^{(4)}=\frac{1}{16}$ for computations with the matrix valued dissipation model. These values of $\kappa$ are 16 times the recommended values in the JST model (\$4.3). As indicated earlier, particular values of the constants may be optimized for a given basic scheme. Here we chose the values of $\kappa$ only to ensure sufficient damping. Parametric studies of the constants may yield a better choice. In addition to different choices of $\kappa$, one may also try to optimize this dissipation model with different choices of switching parameters e.g., $\mu_{i}, \nu_{i}$, etc. in \$4.4. However, fine tuning of the parameters is expensive and may not be always feasible in practical computations. The Tam dissipation model has a scaling parameter known as the stencil Reynolds number $\left(R_{s}\right)$. Effect of this parameter and also a comparison of the standard 4th and the 6th order central difference schemes with the DRP scheme are shown in Figure 3. The DRP scheme which we consider in our tests is formally 4th order accurate in space and uses a seven point stencil. The standard 4th order central difference scheme uses a five point stencil and the standard 6 th order central difference scheme uses a seren point stencil. We observe that the DRP scheme performs better than the standard 4th order scheme. However, the differences between the DRP scheme and the standard 6 th order central difference scheme for our present computations appear to be very small. These results show the same trend as in wave equation computations presented by $\operatorname{Tam}^{17}$. The artificial dissipation may have reduced the differences among the schemes. We also observe that with the change of the stencil Reynolds number $\left(R_{s}\right)$, both the solution profile and the shock location change slightly.

Next we evaluate the dissipation models for computation of the test problem 1 with the fourth order MacCormack scheme. We used the CFL number equal to $\frac{1}{2}$ for all computations in this paper using the fourth order MacCormack scheme. Density profile computed using this scheme without any added artificial dissipation is shown in Figure 4. We also show the computed profile using the DRP scheme augmented with the Tam dissipation model $\left(R_{d}=0.1\right)$. The fourth order MacCormack scheme with its builtin dissipation appears to generate minor spurious oscillations. One may use artificial dissipation model to reduce these oscillations. Effects of various dissipation models on the computed density profiles near the shock are shown in Figure 5. Values of $\kappa$ used in the JST and Turkel's matrix dissipation model are given in $\$ 4.3$. We used higher values of stencil Reynolds number for the Tam dissipation model to avoid excessive smearing of the shock profile. Both MacCormack-Bald win (MB) and Turkel's matrix dissipation model gave sharp shock profiles. Other models also performed reasonably well. The Tam dissipation model appears to be slightly better in eliminating preshock oscillations, but also seems to smear the shock profile more than other models.

Our second test problem has a very sharp gradient in the initial condition. In Figure 6, we show the initial and computed profiles of this test problem at time equal to 50 . Computed solutions were obtained using the DRP and the standard 6th order central difference schemes. In this plotting scale, differences between the DRP and the 6th order central difference scheme are not noticeable. The Tam dissipation model with the stencil Reynolds number equal to 0.1 was used for these computations. There are minor oscillations near the shock. We concentrate on that region in the next two figures. In Figure 7, we compare solutions with the DRP scheme with and without artificial dissipation. Model constants $\kappa^{(2)}$ and $\kappa^{(4)}$ in the matrix dissipation were kept as in the case shown in Figure 2. Both the Tam dissipation and Turkel's matrix dissipation models were effective in eliminating spurious oscillation in the computed solutions. The Tam dissipation model with the stencil Reynolds number $\left(R_{s}\right)$ equal to 0.05 appears to be slightly better that the recommended value i.e., 0.1 in removing most of the oscillations. The shock location is slightly different with these two values of the stencil Reynolds numbers. We then compare the DRP and the standard central difference schemes with the Tam dissipation model in Figure 8. Differences in the computed solutions with the DRP and the standard central difference schemes appear to be small. This may be partially caused by the presence of large amount of artificial dissipation in the computed solutions. Next we examine the dissipation models in the MacCormack scheme for computation of the test problem 2 at time equal to 50. In Figure 9, we show the density profile computed using the MacCormack scheme withont dissipation and compare it with the solution using the DRP scheme with the Tam dissipation model $\left(R_{t}=0.1\right)$. The MacCormack scheme without dissipation generates oscillations near the shock. We examined the effect of various dissipation models on the MacCormack scheme in Figure 10. The shock profile is slightly sharper with the matrix dissipation model than other models. As in the 
case with the test problem 1, preshock oscillations were slightly less with the Tam dissipation model, but the shock profile was slightly more smeared. We examine the profile near the contact discontinuity in Figure 11. For computations in this region, MacCormack-Bald win (MB) and Turkel's matrix dissipation model performed well.

Our last test problem is a shock tube problem and was used by Lax ${ }^{21}$. In this paper we show computed solutions of this problem at time equal to 0.16 . For this model problem, the values of $\kappa$ used in the matrix dissipation model are 16 times the recommended values of the JST model (\$4.3). This choice was made only to ensure sufficient artificial dissipation for the test cases without any optimization of the values of $\kappa$. As shown in Figure 12, major differences in computed solutions are limited to regions around the discontinuities. We focus on this region for computations with the DRP and MacCormack schemes in next two figures. In Figure 13 we compare computed density profiles using the DRP scheme with the exact solution. The computed profile with the stencil Reynolds number equal to 0.1 appear to be in reasonable agreement with the exact solution. Solution using the fourth order DRP scheme (seven point stencil) is very close to that using the standard sixth order central scheme (seven point stencil). For the stencil Reynolds number equal to $\mathbf{0 . 0 5}$, the solution is smeared and the shock location is slightly shifted. The smearing of the shock profile is due to excessive amount of artificial disspation. The change in the shock location may in part be caused by the Tam dissipation model which is not cast in conservation form. Tam and Shen ${ }^{2}$, however, pointed out that some dispersion error may come from their damping model. We next examine computations using the fourth order MacCormack Scheme. Solutions using this scheme are shown in Figure 14. As in the case of previous test problems, computed solutions without any artificial dissipation exhibit oscillations. We also show the results with the MacCormack-Bald win and Turkel's matrix disspation model in this figure. Artificial dissipation models eliminated most of the spurious numerical oscillations. Our choices of the values of $\kappa$ may have caused excessive damping with the matrix dissipation model. Finally we show computed pressure profiles of Lax's problem in Figures 15 and 16. Here again we find that artificial dissipations eliminated most of the oscillations in the DRP (see figures 2, 7 and 8 for solutions asing the DRP scheme without artificial dissipation) and the fourth order MacCormack scheme without artificial dissipation. Basic observations are essentially the same as from computed density profiles.

In summary, we would like to say that various artificial models considered in this study in general performed well. However, one probably should optimize some parameters to improve the quality of the solutions. The fourth order MacCormack scheme without any artificial dissipation generates spurious oscillations near sharp gradients. We recommend adding artificial dissipation for computations of nonlinear waves or flows with shocks with this scheme. Artificial dissipation plays a more important role in getting good computed solutions using the DRP scheme. Without any artificial damping, the DRP scheme appears to generate excessive numerical oscillations.

\section{Acknowledgements}

The author would like to thank Dr. Hung T. Huynh of NASA Lewis for providing him the exact solution of Lax's problem. He would also like to thank Dr. Philip Jorgenson of NASA Lewis for explaining the details of the matrix dissipation model. This work was supported by NASA Cooperative Agreement NCC3-233 through the ICOMP program.

\section{References}

${ }^{1}$ Tam, C. K. W. and Webb, J. C., (1993), "Dispersion- Relation- Preserving Finite Difference Schemes for Computational Acoustics", Journal of Computational Physics, 107, Pp 262-281.

${ }^{2}$ Tam, C. W. K. and Shen, H., (1993), "Direct Computation of Nonlinear Acoustic Pulses Using High Order Finite Difference Schemes", AIAA paper 93-4325.

${ }^{3}$ Tam, C.. K. W., Webb, J. C, and Dong, Z., (1993), "A Study of the Short Wave Components in Computational Acoustics", Journal of Computational Acoustics, Vol 1, No 1, pp 1-30. 
${ }^{4}$ Gottlieb, D. and Turkel, E., (1976), "Dissipative Two-Four Methods for Time Dependent Problems”, Math. Comp, Vol 30, pp 703-723.

${ }^{5}$ Bayliss, A., Maestrello, L., Parikh, P. and Turkel, E., (1985), "A Fourth Order Method for the Unsteady Compressible Navier-Stokes Equations," AIAA paper 85-1694.

'Sankar, L. N., Reddy, N. N. and Hariharan, N., (1993), "A Comparative Study of Numerical Schemes for Aero-Acoustic Applications," FED Vol. 147, Computational Aero- and HydroAcoustics, ASME 35-40.

${ }^{7}$ Farouk, B., Oran, E. S. and Kailasanath, K., (1991) "Numerical Simulations of the Structure of Supersonic Shear Layers," Physics of Fluids A, 3, 2786-2798.

${ }^{8}$ Frendi, A., Maestrello, L. and Bayliss, A., (1994), "Coupling between Plate Vibration and Acoustic Radiation," Journal of Sound and Vibration, 177, 207-226.

${ }^{9}$ Ragab, S. A. and Sheen, S., (1991), "The Nonlinear Development of Supersonic Instability Waves in a Mixing Layer," Physics of Fluids A, 4, 553-566.

${ }^{10}$ Hayder, M. E., Turkel, E. and Mankbadi, R. R., (1993), "Numerical Simulations of a High Mach Number Jet Flow", AIAA paper 93-0653, NASA TM 105985.

${ }^{11}$ Jameson, A., Schmidt, W. and Turkel E., (1981), "Numerical Simulations of the Euler Equations by Finite Volume Methods, Using RungeKutta Time-Stepping Schemes", AIAA Paper 811259.

${ }^{12}$ Turkel, E., (1988). "Improving the Accuracy of Central Difference Schemes", ICASE report 8853.

${ }^{13}$ MacCormack, R. W. and Baldwin, B. S., (1975), "A Numerical Method for solving the Navier Stokes Equations with Application to Shock Boundary layer Interactions", AIAA paper 75-1.
${ }^{14}$ Lax, P., D. and Wendroff, B., (1960), "System of Conservation Laws", Comm. Pure and Applied Mathematics, 13, pp 217-237.

${ }^{15}$ Hirsch, C., (1988), "Numerical Computation of Internal and External Flows", Vol 1, John Wiley \& Sons.

${ }^{16}$ Hayder, M. E. and Turkel, E. (1993), "High Order Accurate Solutions of Viscous Problems", AIAA paper 93-3074, NASA TM 106267.

${ }^{17}$ Tam, C. K. W., (1995), "Computational Aeroacoustics: Issues and Methods", AIAA paper 95-0677.

${ }^{18}$ Anderson, D. A., Tannehill, J. C. and Pletcher, R. H., (1984), "Computational Fluid Mechanics and Heat Transfer", McGraw-Hill Book Company.

${ }^{19}$ Jorgenson, P. and Turkel, E., (1993), "Central Difference TVD Schemes for Time Dependent and Steady State Problems", Journal of Computational Physics, 107, pp 297-308.

${ }^{20}$ Hardin, J. C. ed. Computational Aeroacoustics Benchmark Problems, Proceedings of the ICASE/LaRC Workshop on Benchmark Problems in Computational Aeroacoustics, Hampton, VA, October 24-26, 1994.

${ }^{21}$ Lax, P., D., (1954), "Weak Solutions of Nonlinear Hyperbolic Equations and Their Numerical Computations", Comm. Pure Appl. Math, 7, pp 159-193.

${ }^{22}$ Hayder, M. E., (1994), "Evaluation of High Order Schemes for Nonlinear Wave Computations", Presented in the ICASE/LaRC Workshop on Benchmark Problems in Computational Aeroacoustics, Hampton, VA, October 1994. 


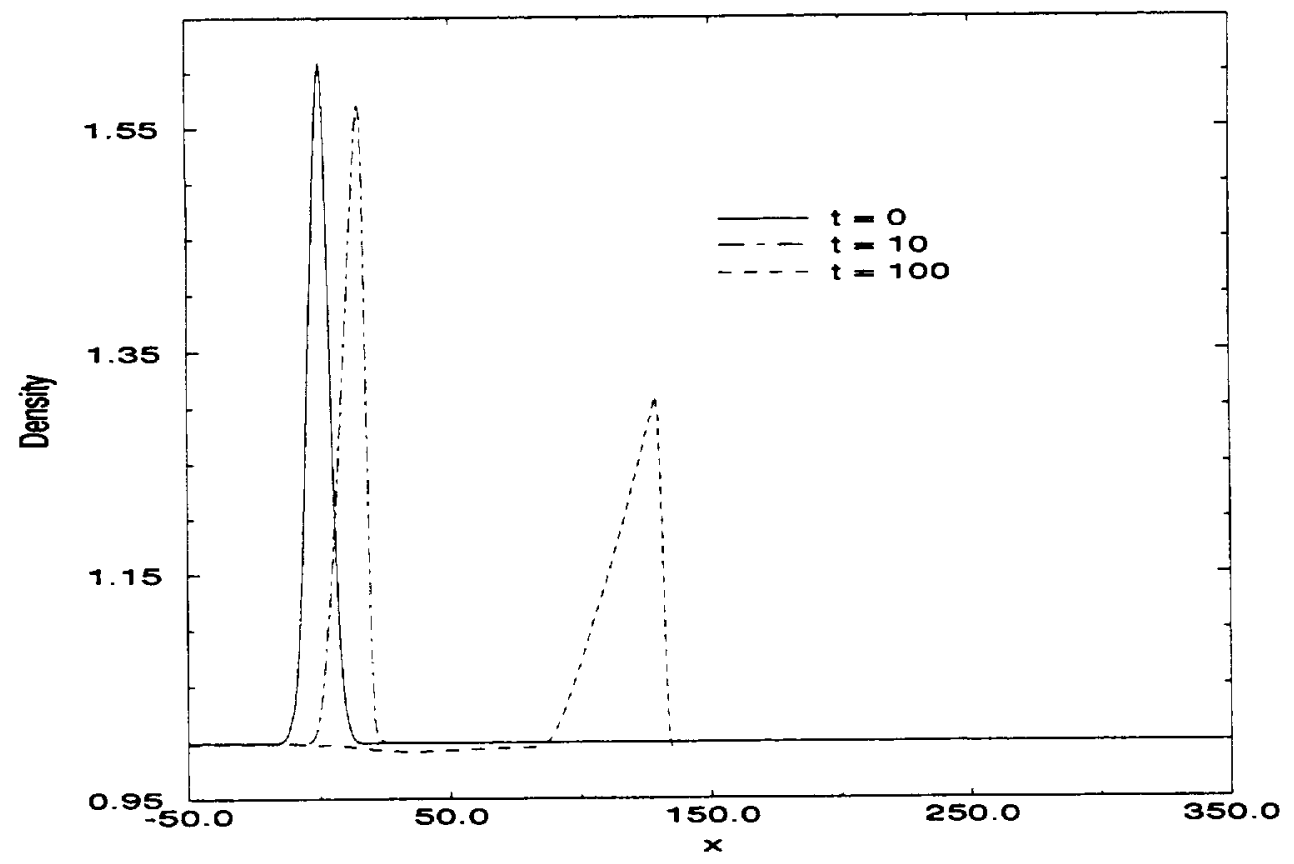

Figure 1: Evolution of the density pulse in problem 1

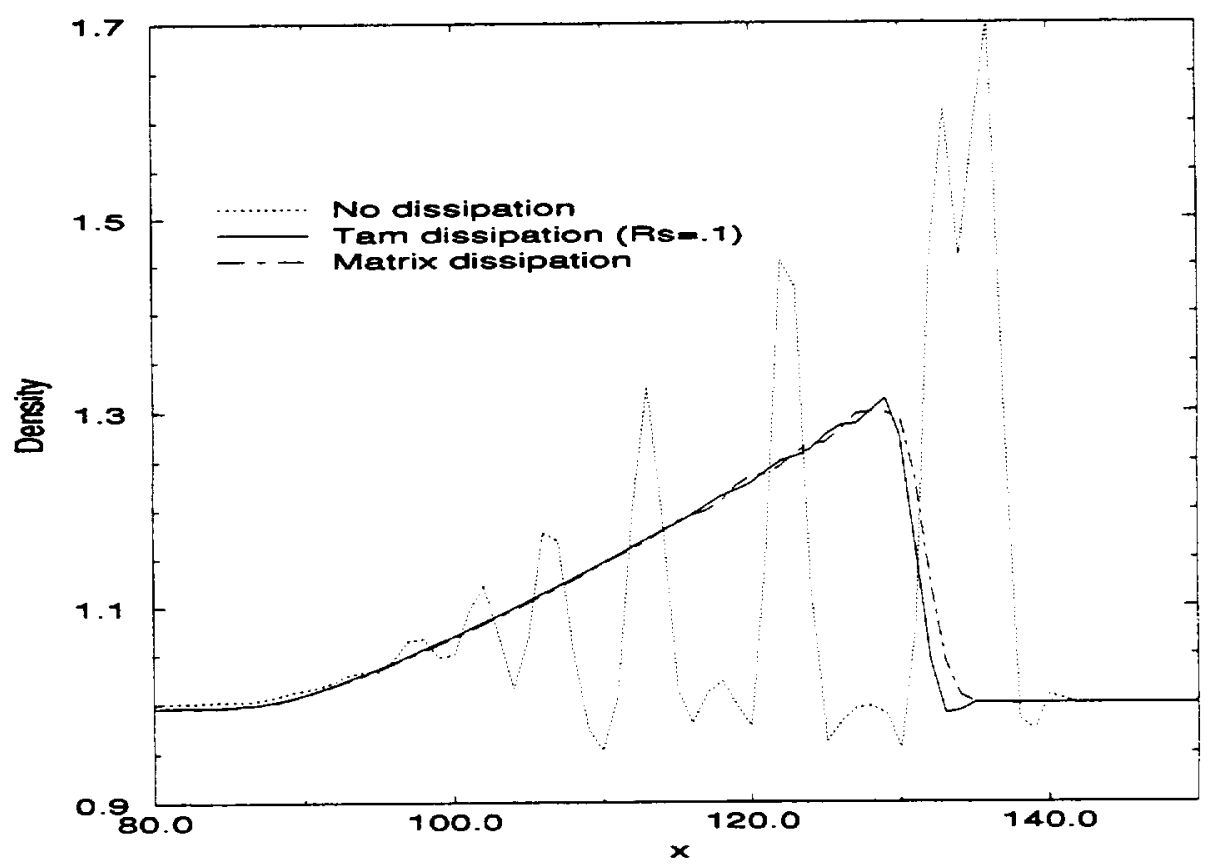

Figure 2: Effect of dissipation on the DRP scheme for problem 1 


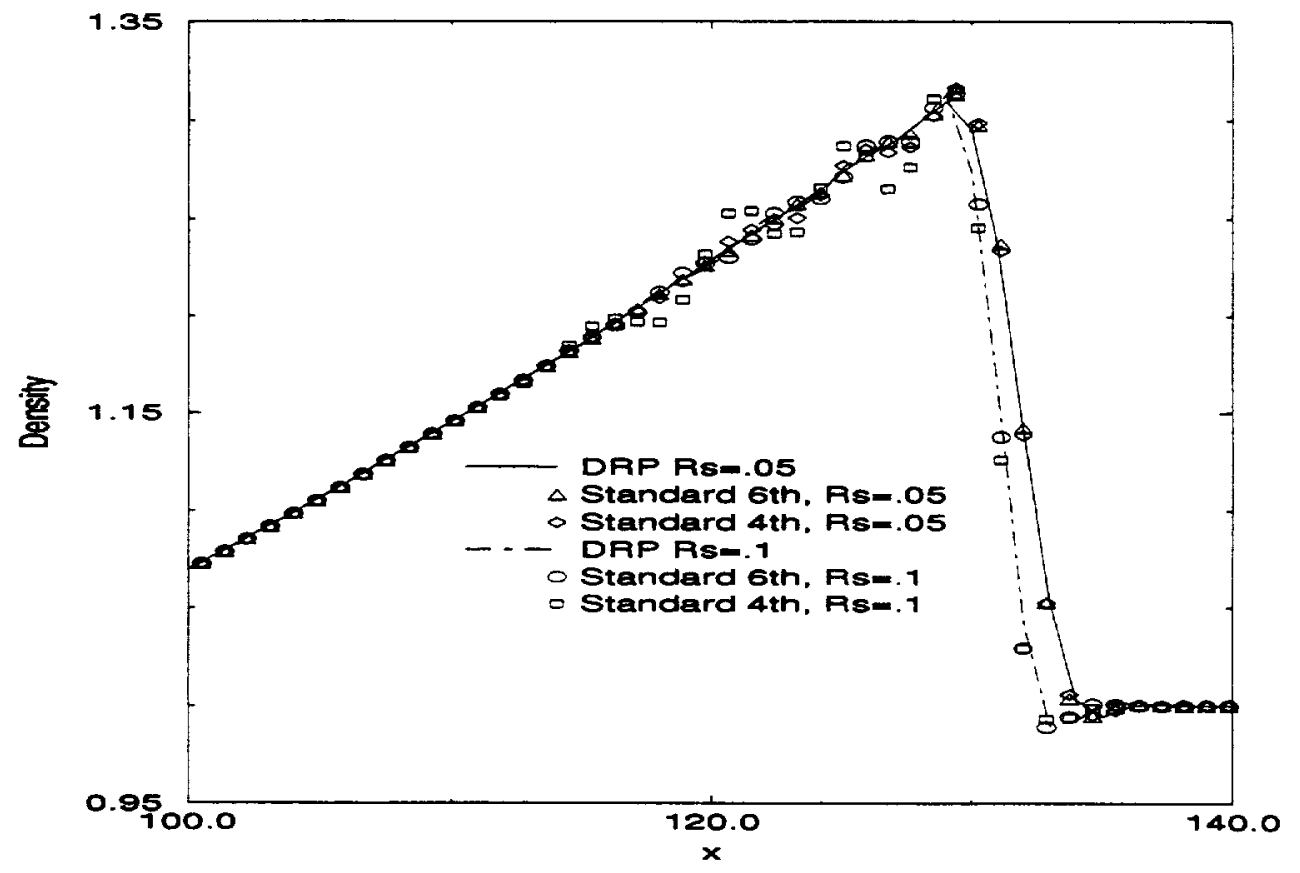

Figure 3: Comparison of central schemes using the Tam dissipation model for problem 1

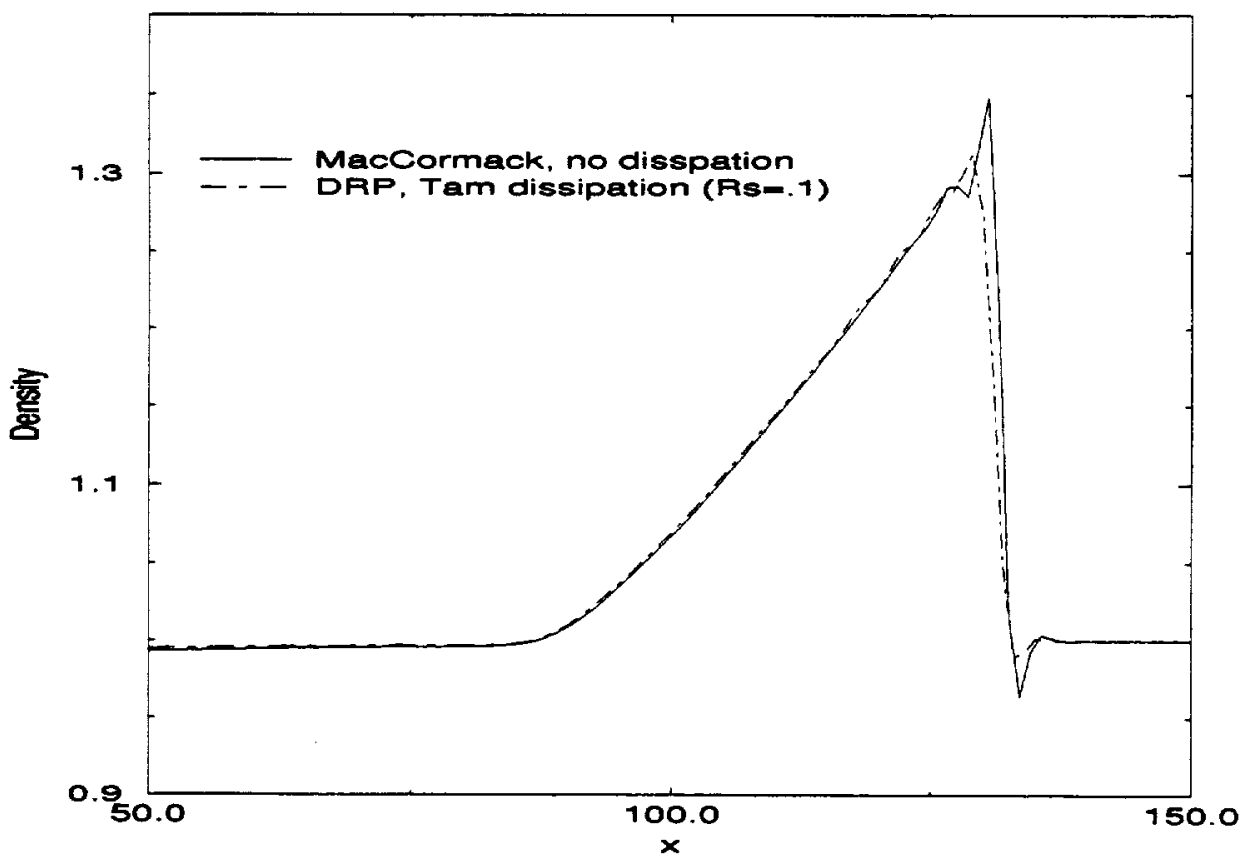

Figure 4: Comparison of the DRP and the MacCormack schemes for problem 1 


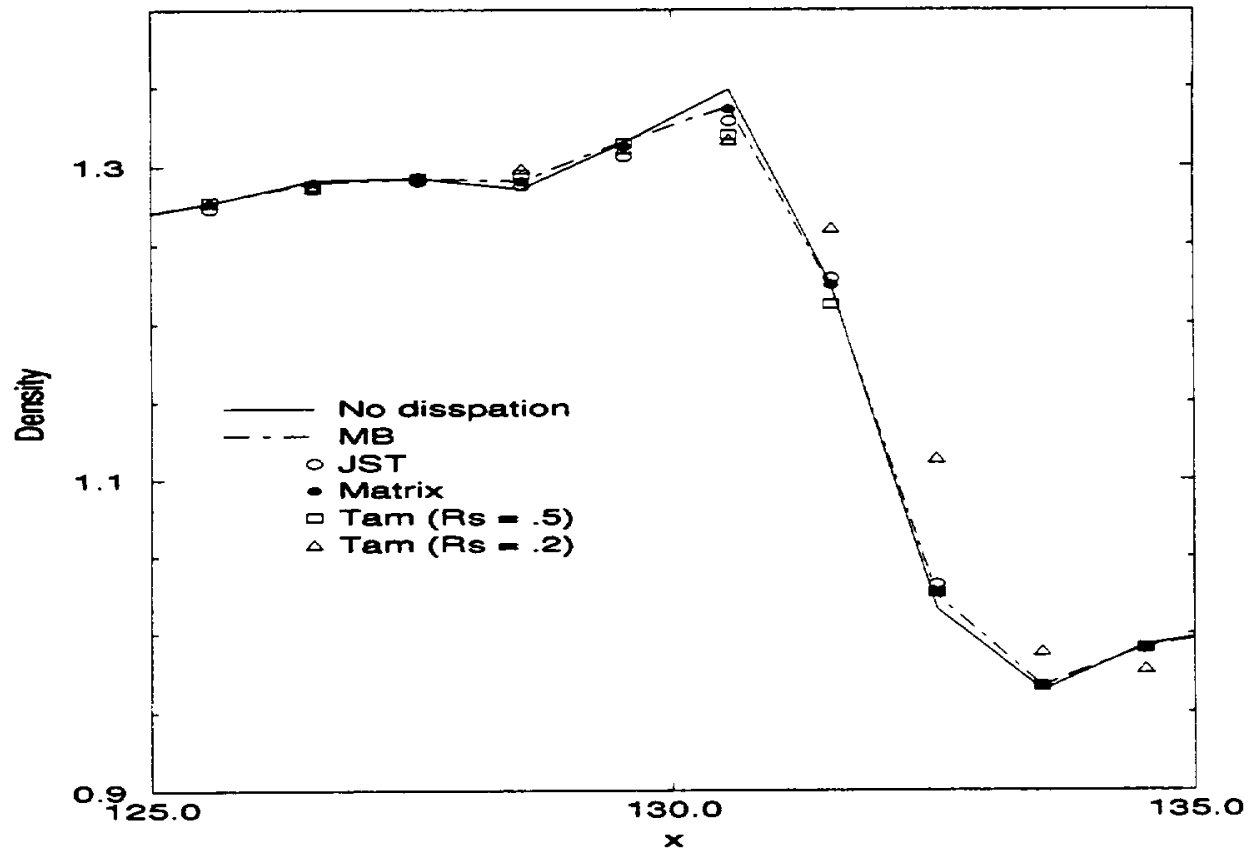

Figure 5: Effect of artificial dissipation on the MacCormack scheme for problem 1

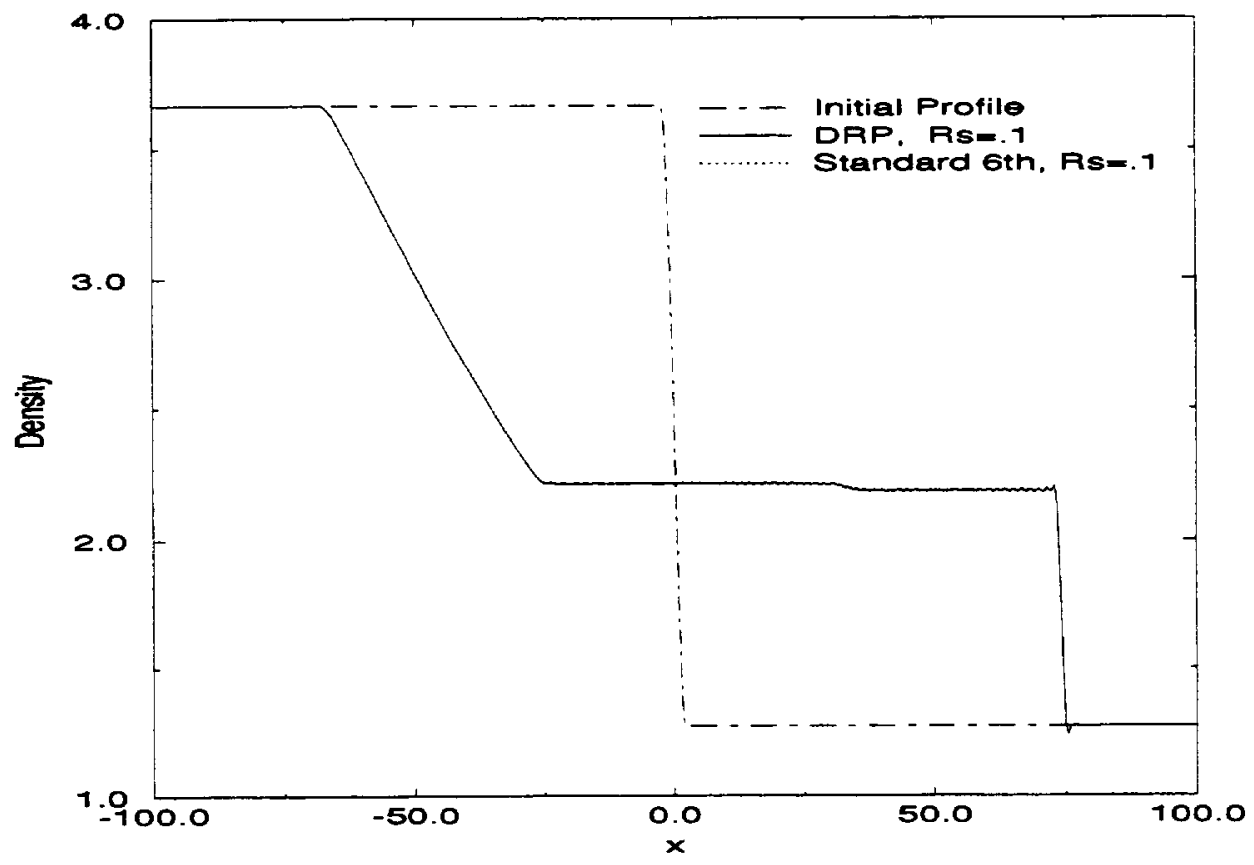

Figure 6: Evolution of density profile in problem 2 


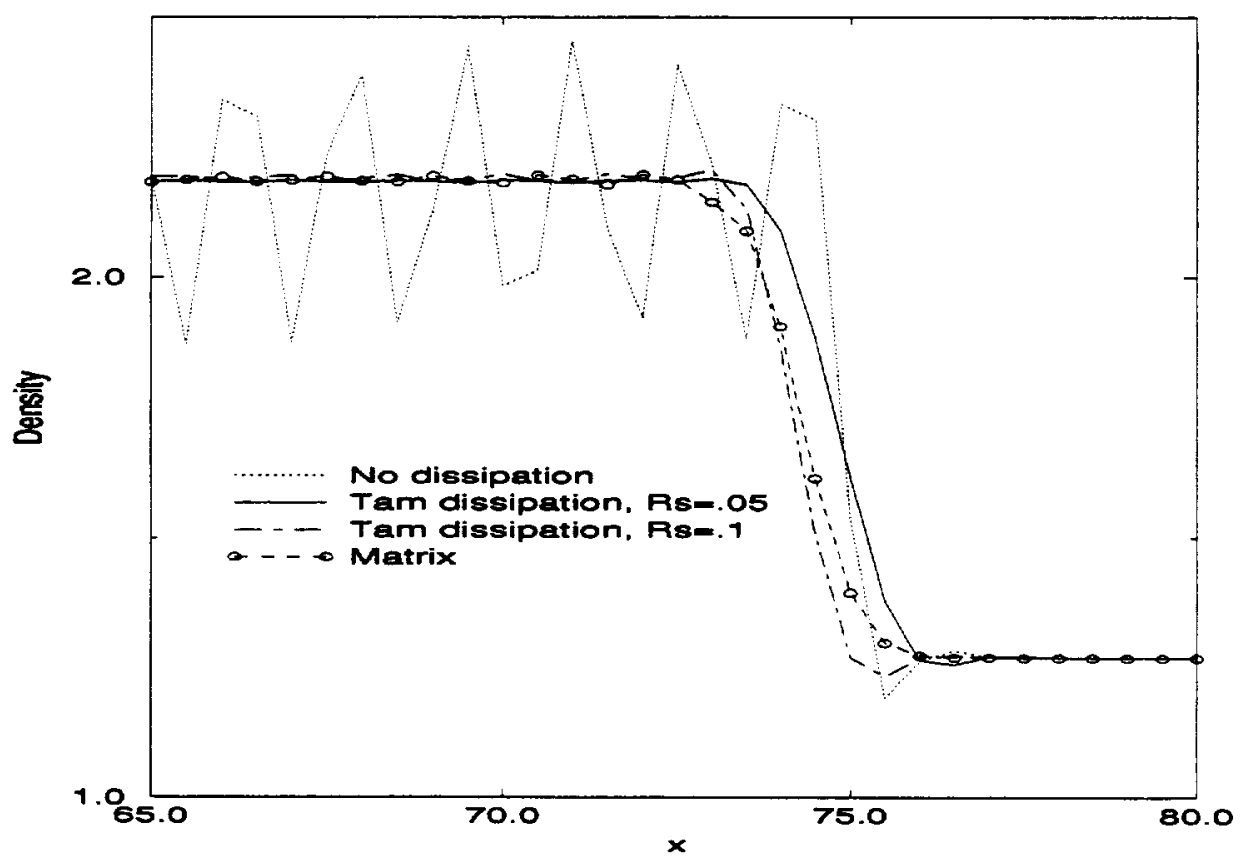

Figure 7: Effect of artificial dissipation on the DRP scheme for problem 2

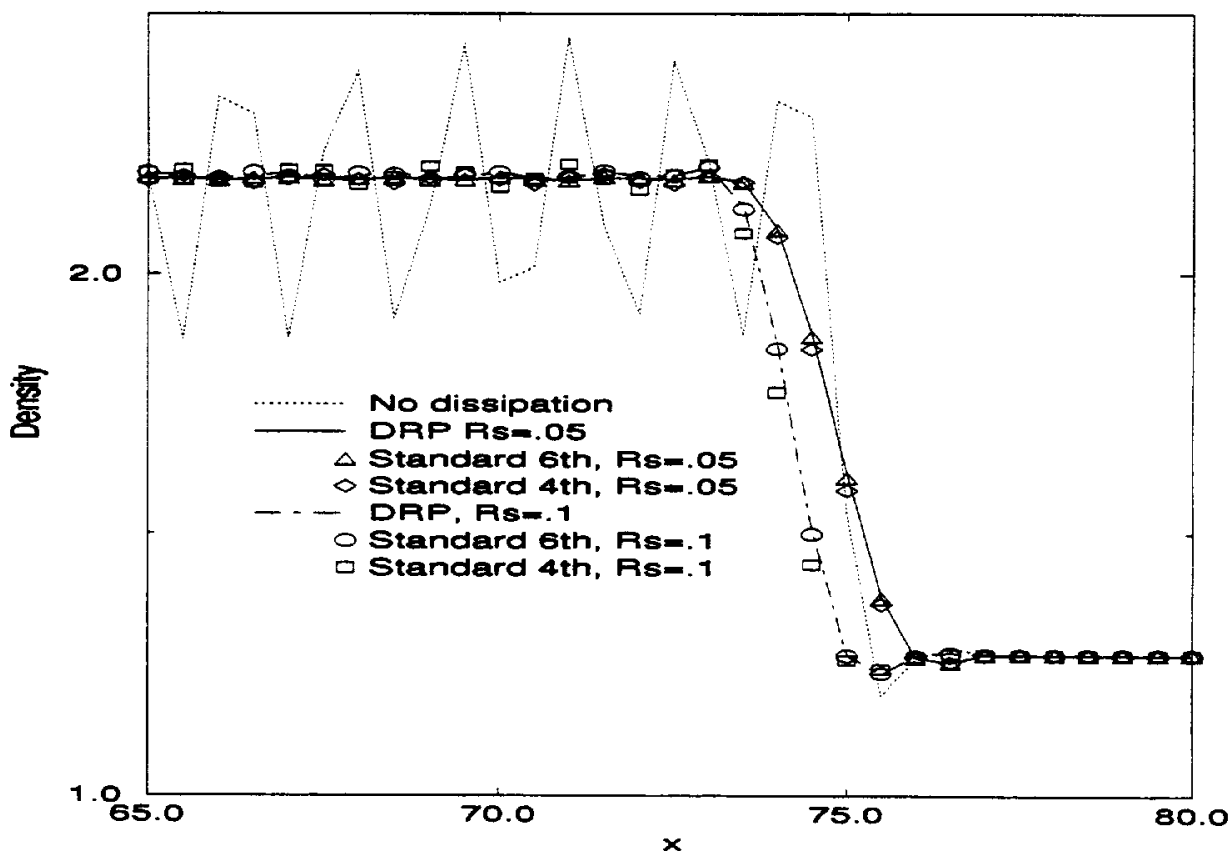

Figure 8: Comparison of central schemes using the Tam dissipation model for problem 2 


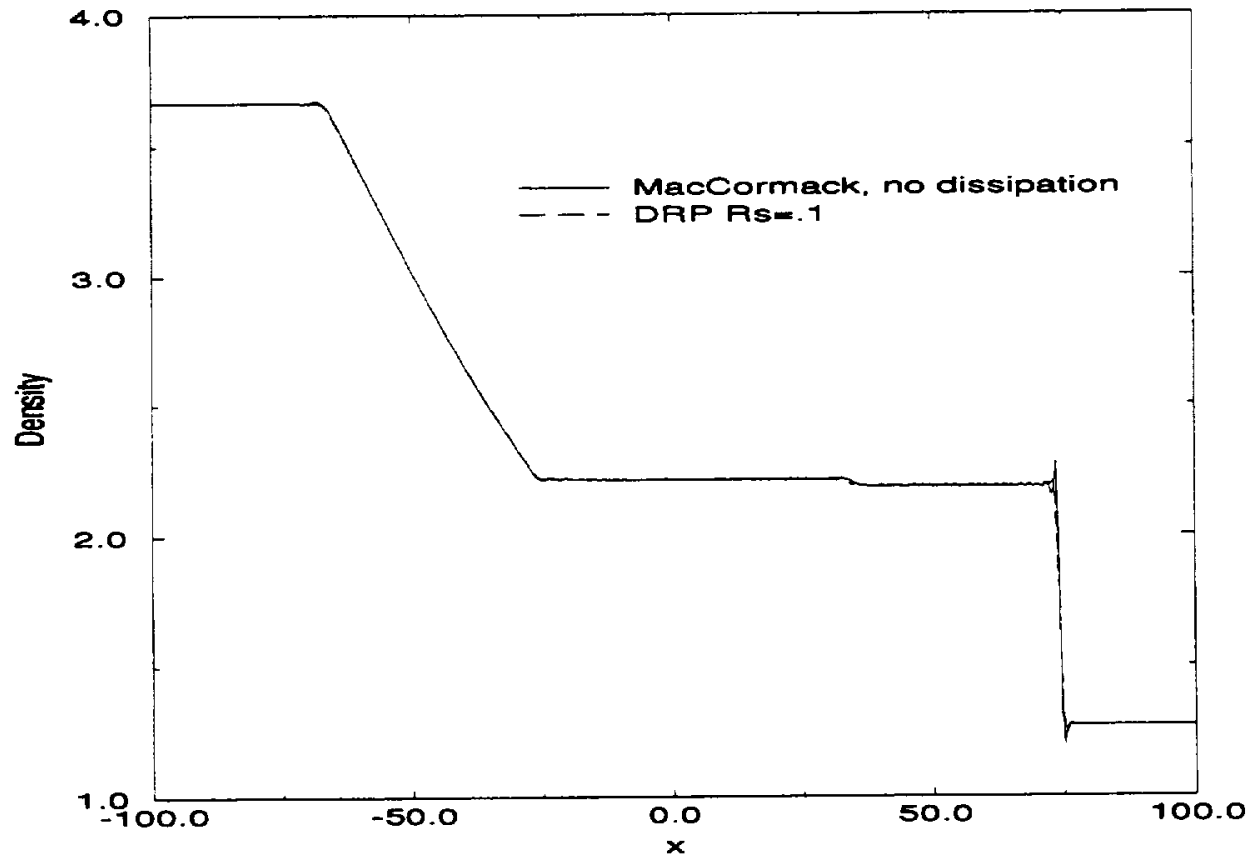

Figure 9: Comparison of the DRP and the MacCormack schemes for problem 2

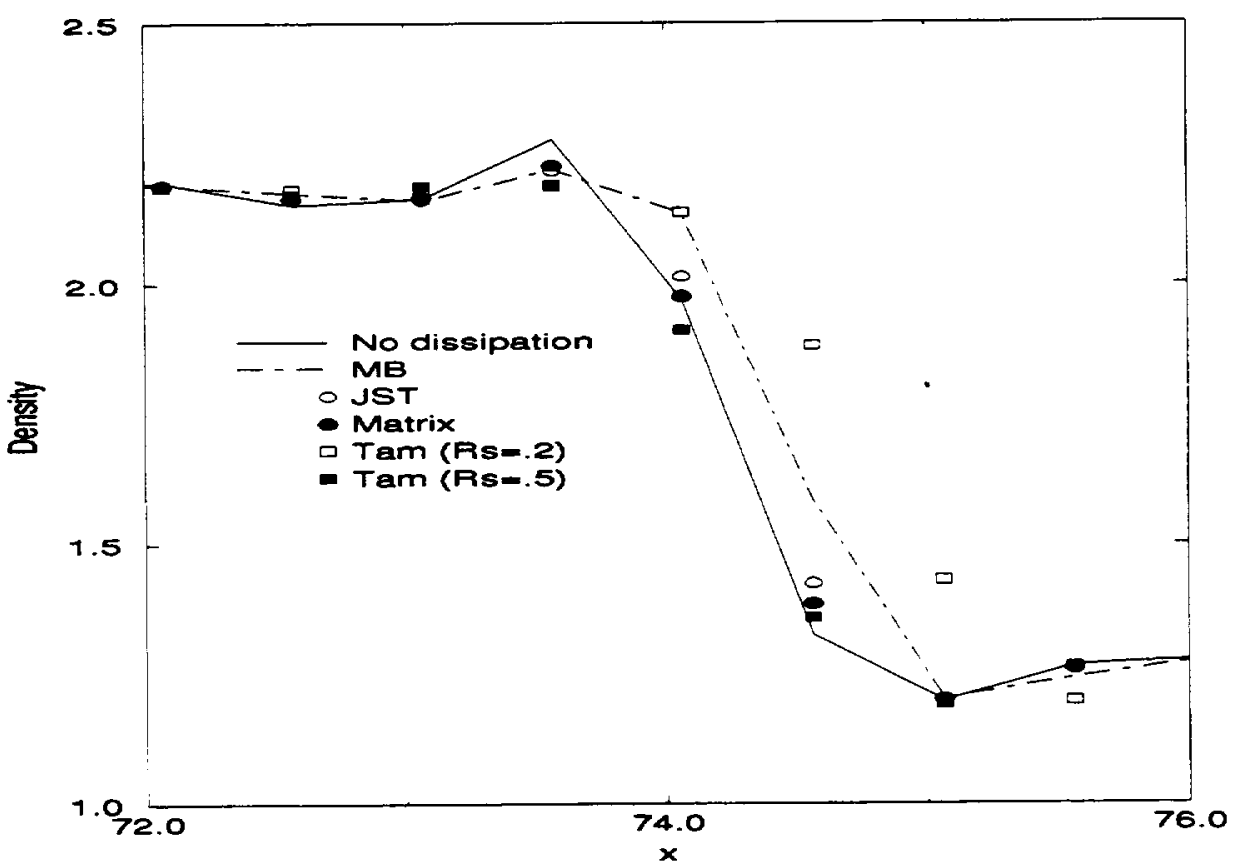

Figure 10: Shock profiles computed by the MacCormack scheme for problem 2 


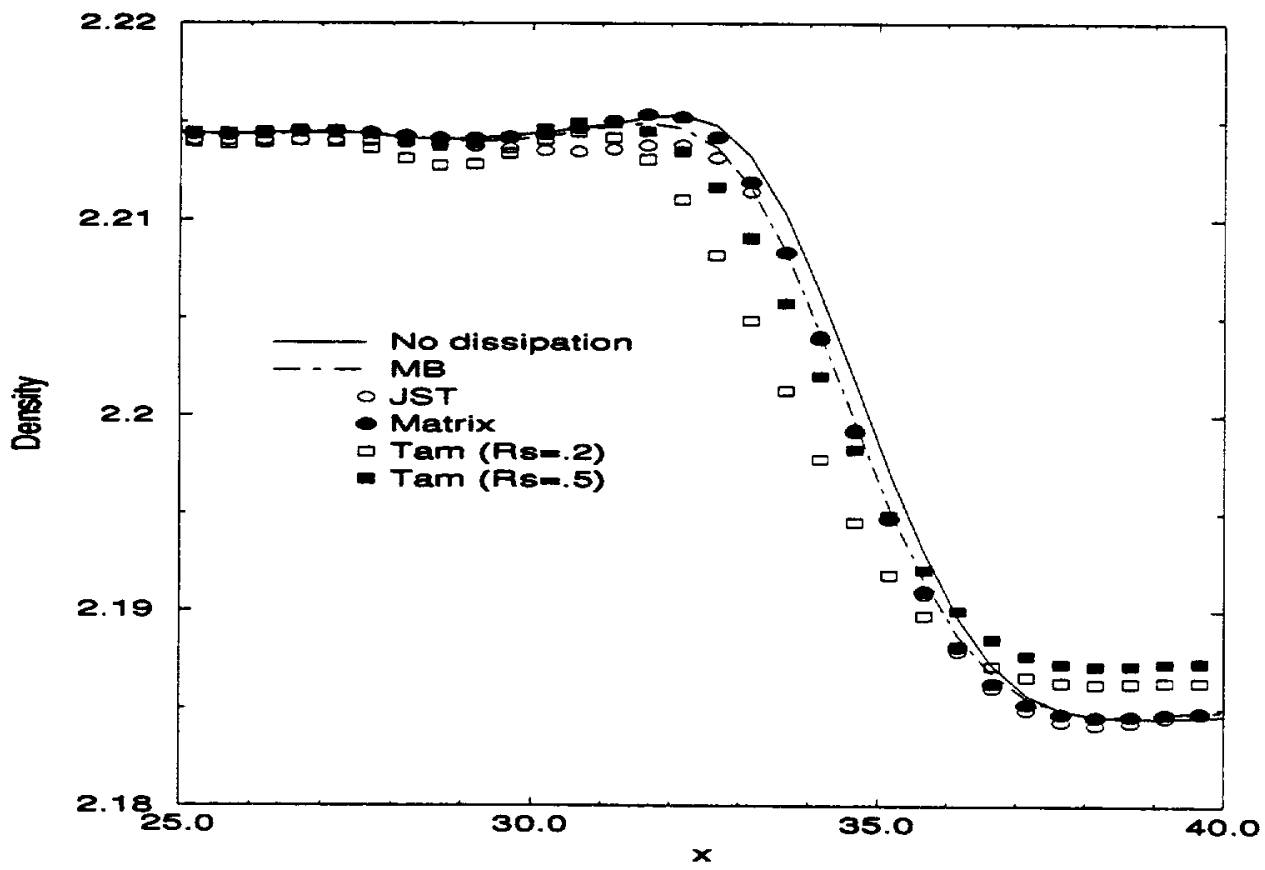

Figure 11: Computed contact discontinuities using the MacCormack scheme for problem 2

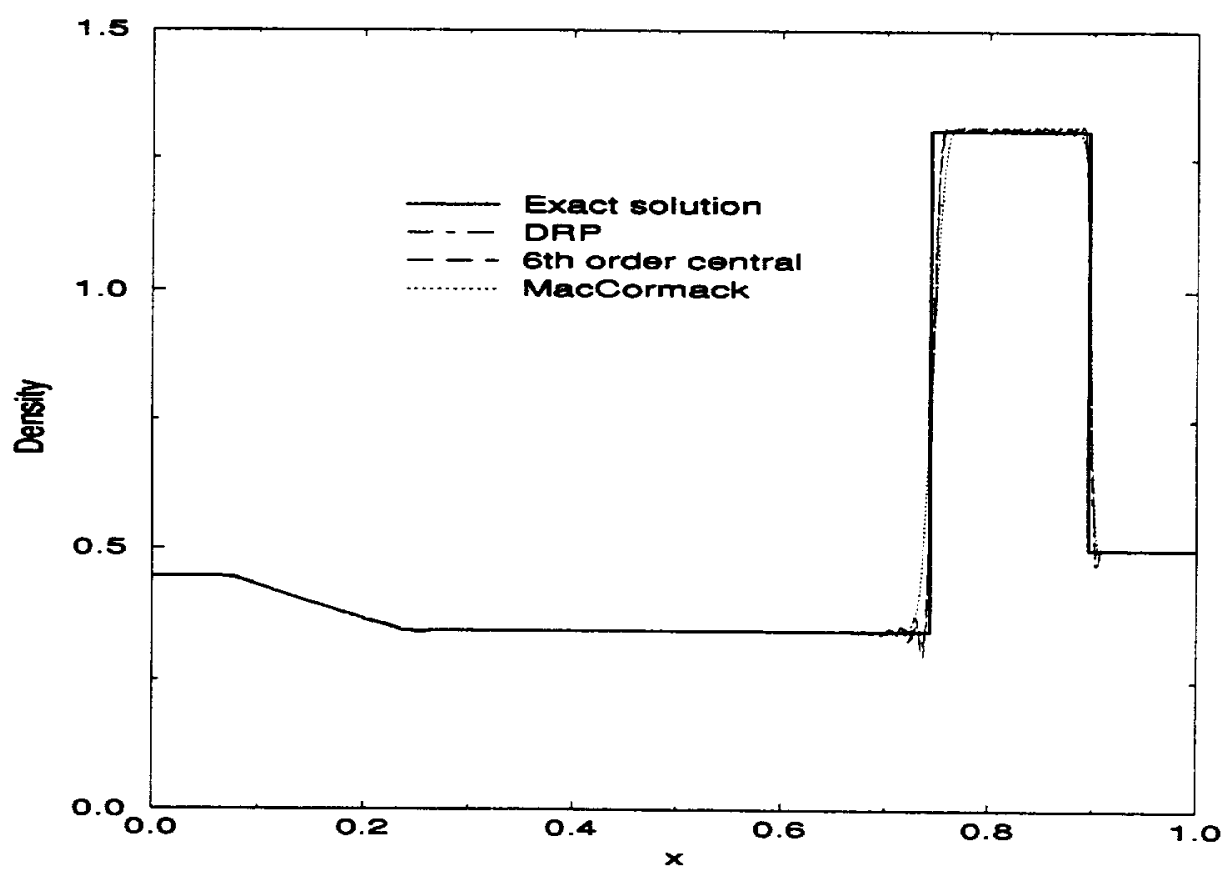

Figure 12: Computed density profile of Lax's problem 


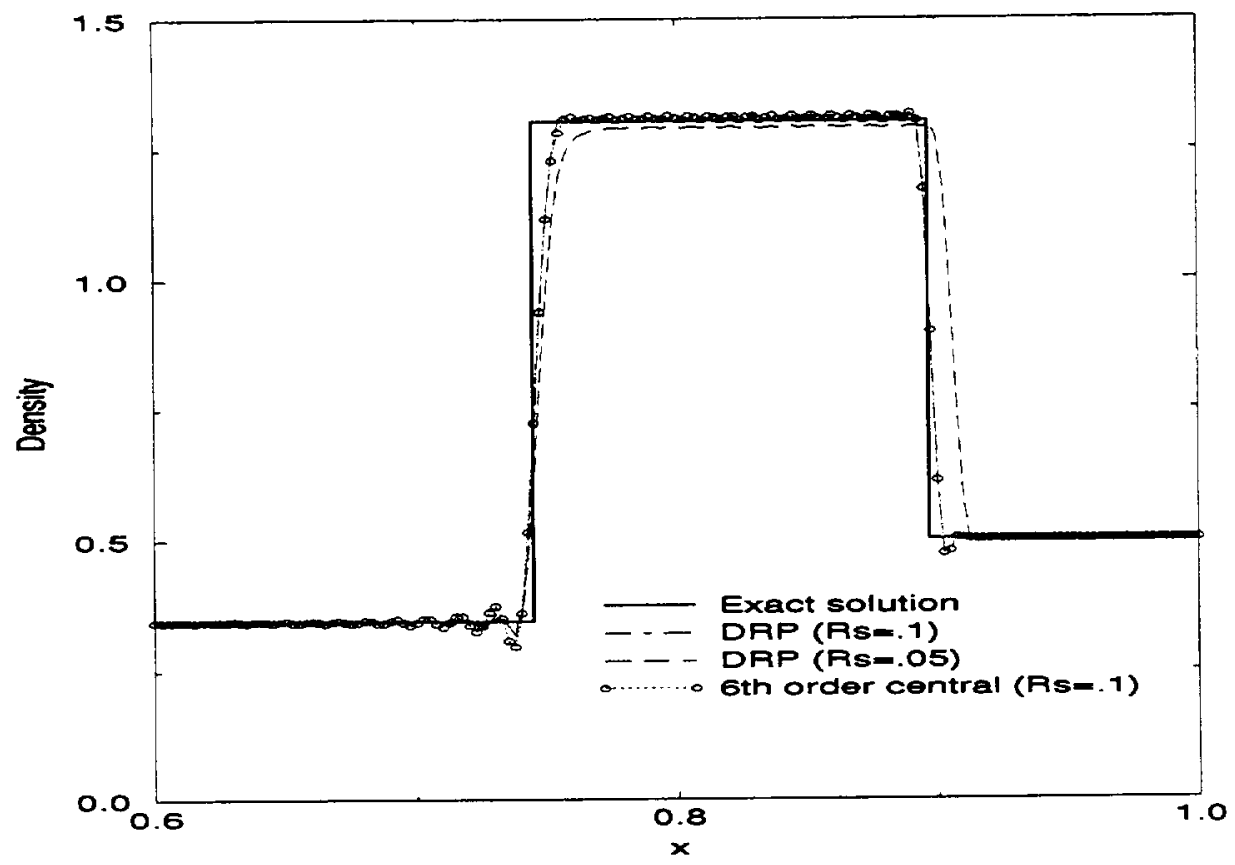

Figure 13: Computation of Lax's problem by the DRP scheme

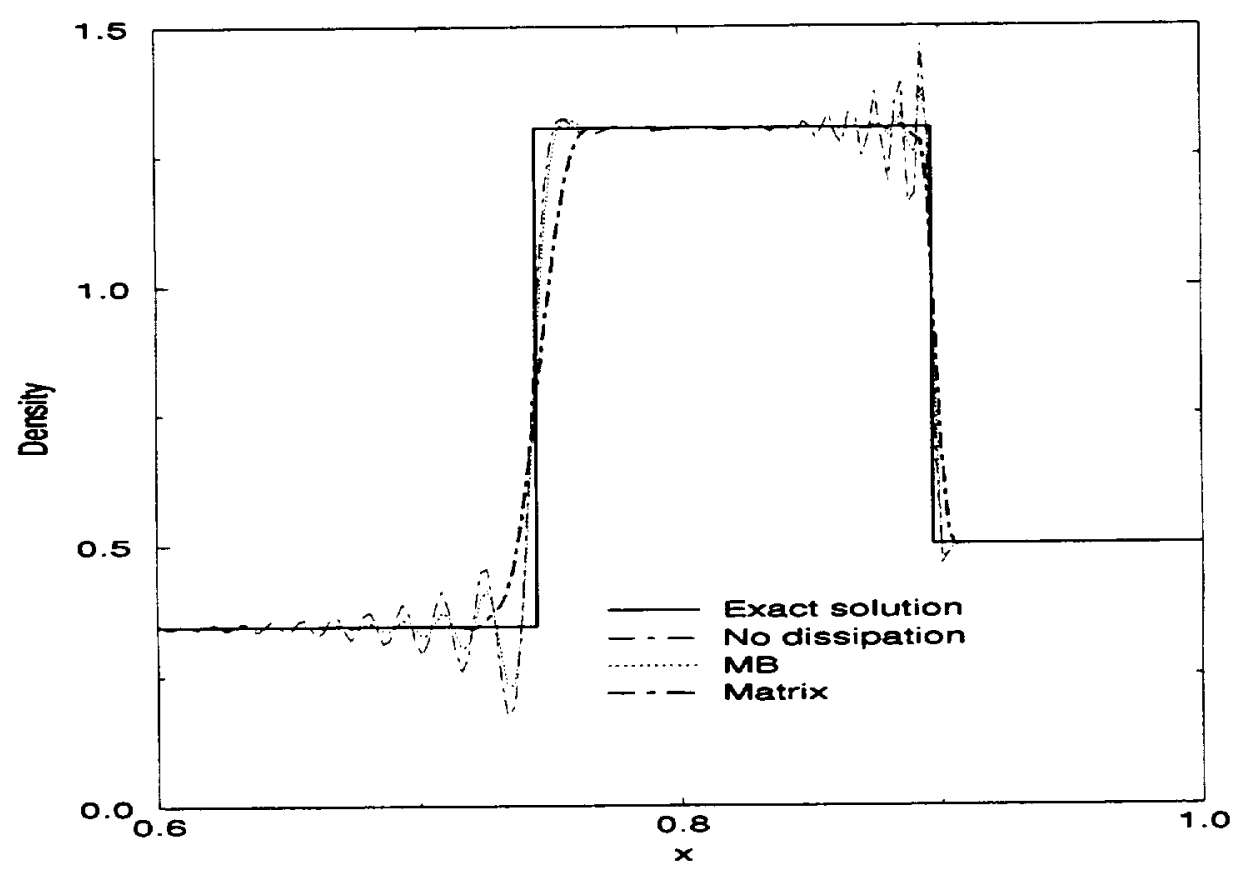

Figure 14: Computation of Lax's problem by the MacCormack scheme 


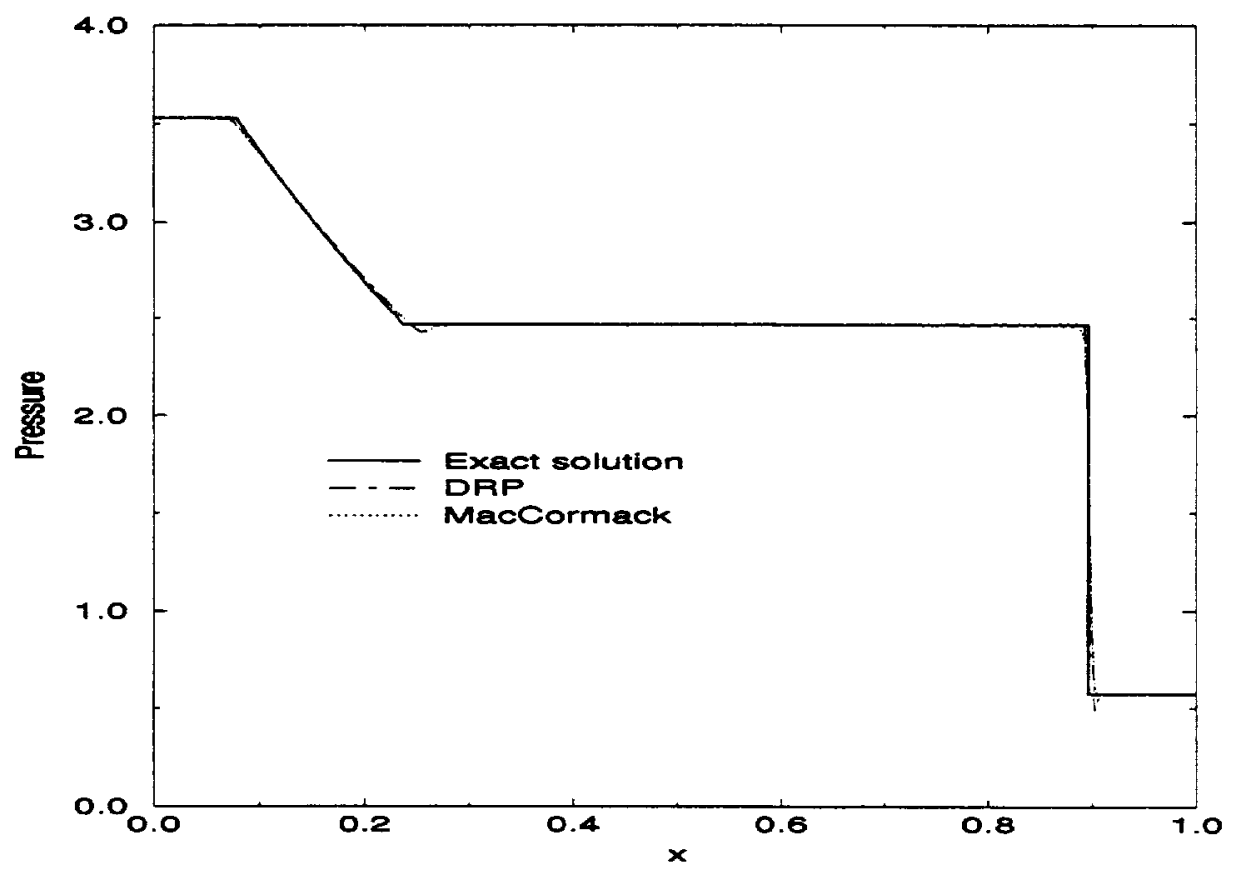

Figure 15: Computed pressure profile of Lax's problem

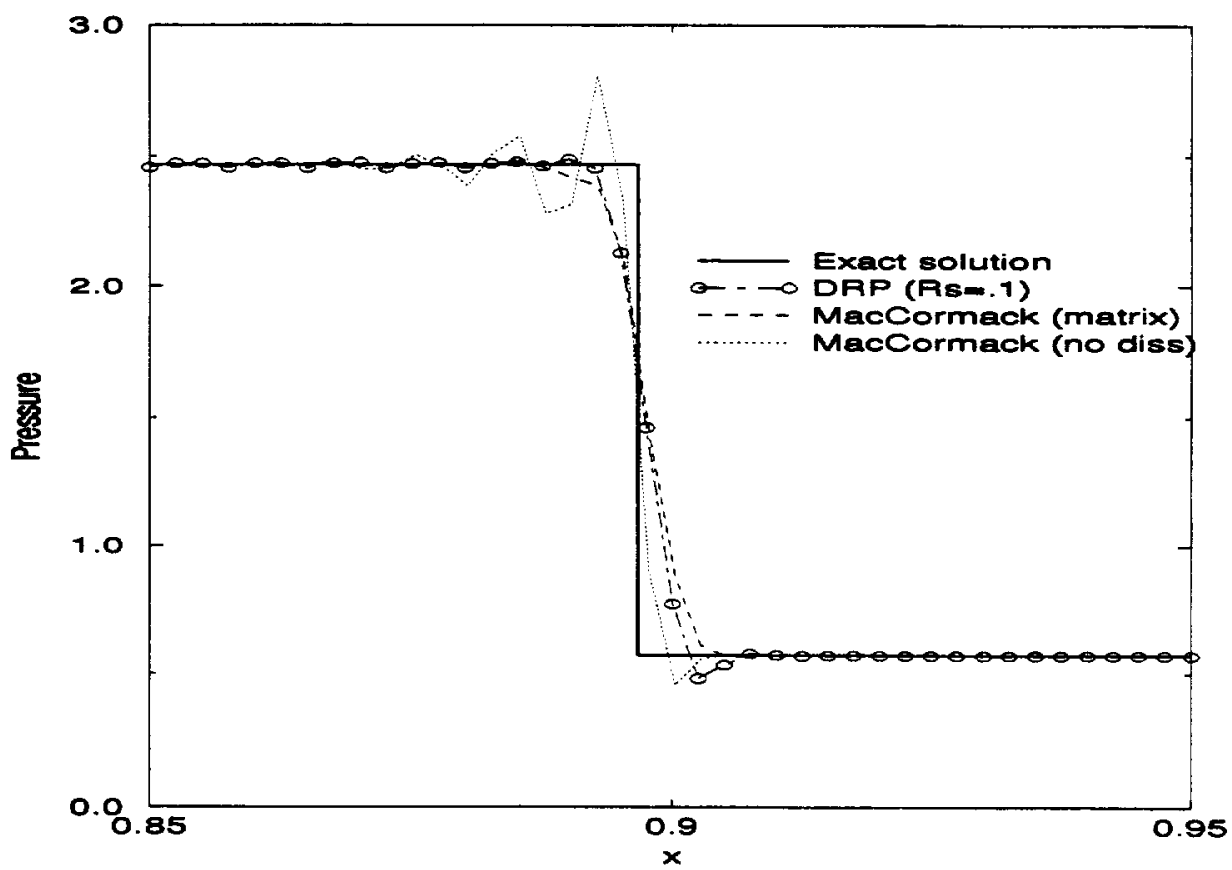

Figure 16: Computed pressure profile near the shock 


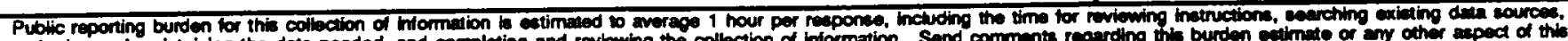

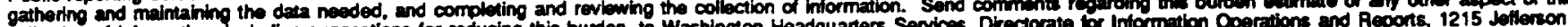
collection of information, hncluding suggestions for reducing this burden, to Washington Headquarters Services, Directorate

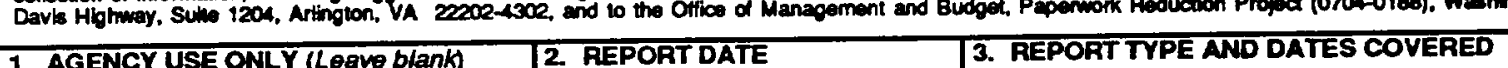

1. AGENCY USE ONLY (Leave blank)
$\begin{aligned} & \text { 2. REPORT DATE } \\ & \text { June } 1995\end{aligned}$

4. TITE AND SUBTILE

5. FUNDING NUMBEAS

An Assessment of Artificial Damping Models for Aeroacoustic Calculations

6. AUTHOR(S)

WU-505-90-5K

NCC3-370

M. Ehtesham Hayder

7. PERFORMING ORGANIZATION NAME(S) AND ADDRESS(ES)

8. PERForiming ORGAMIZATION REPORT NUMBER

Institute for Computational Mechanics in Propulsion

Lewis Research Center

E-9728

Cleveland, Ohio 44135-3191

9. SPONSORINGMONITORING AGENCY NAME(S) AND ADDRESS(ES)

National Aeronautics and Space Administration

Lewis Research Center

Cleveland, Ohio 44135-3191

10. SPONSOAINGNONITORING

AGENCY REPOAT NUMBER

NASA CR-198354

ICOMP-95-11

AIAA-95-2167

11. SUPPLEMENTARY NOTES

Prepared for the 26th Fluid Dynamics Conference sponsored by the American Institute of Aeronautics and Astronautics,

San Diego, California, June 19-22, 1995. ICOMP Program Director, Louis A. Povinelli, organization code 2600, (216)

433-5818.

12a. DISTRIBUTIONAVALABILTY STATEMENT

12b. DISTRIBUTION CODE

Unclassified - Unlimited

Subject Categories 34 and 64

This publication is available from the NASA Center for Aerospace Information, (301) 621-0390.

13. ABSTRACT (Maximum 200 wonds)

We present a study of the effect of artificial dissipation models on nonlinear wave computations using a few high order schemes. Our motivation is to assess the effectiveness of artificial dissipation models for their suitability for aeroacoustic computations. We solve three model problems in one dimension using the Euler equations. Initial conditions are chosen to generate nonlinear waves in the computational domain. We examine various dissipation models in central difference schemes such as the Dispersion Relation Preserving (DRP) scheme and the standard fourth and sixth order schemes. We also make a similar study with the fourth order MacCormack scheme due to Gottieb and Turkel.

14. SUBJECT TERMS

Artificial dissipation; High order schemes; Computational aeroacoustics

15. NUMBER OF PAGES 18 16. PRICE CODE A03

\begin{tabular}{l|c}
\hline 17. SECURITY CLASSIFICATION & $\begin{array}{c}\text { 18. SECURTY CLASSIFICATION } \\
\text { OF REPORT } \\
\text { OF THIS PAGE } \\
\text { Unclassified }\end{array}$ \\
Unclassified
\end{tabular}

NSN 7540-01-280-5500
19. SECURTYY CLASSIFICATION OF ABSTRACT Unclassified
20. LMATTATION OF ABSTRACT

Standard Form 298 (Rev. 2-89) Prescrbed by ANSI Sid. Z39-18 298-102 



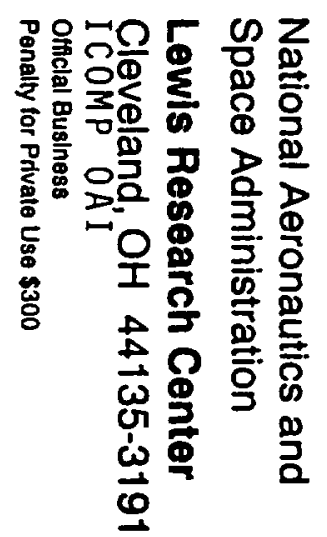


